\title{
Evaluación de la diversidad de bacterias lácticas y levaduras en quesos frescos de cabra de la quebrada de Humahuaca
}

\author{
Ancasi,EG ${ }^{1}$; Maldonado,S ${ }^{1}$; Oliszewski, R. ${ }^{2}$
}

${ }^{1}$ Facultad de Ciencias Agrarias. Universidad Nacional de Jujuy. San Salvador de Jujuy. Jujuy. Argentina.

${ }^{2}$ CONICET - Fac. Agronomía y Zootecnia. Universidad Nacional de Tucumán. Tucumán. Argentina.

\section{Resumen}

Los quesos frescos de cabra artesanales de la quebrada de Humahuaca son elaborados con leche cruda, cuya maduración genera sabores, aromas y texturas característicos de la región. Los objetivos de este estudio fueron identificar y caracterizar bacterias lácticas (BAL) y levaduras nativas, aisladas de quesos frescos de esta zona productora. De un total de 36 muestras sembradas en agar Sabouraud, agar MRS y M17, se obtuvieron 128 levaduras y 39 lactobacilos, los que fueron identificados fenotípicamente y evaluadas las siguientes propiedades tecnológicas: $\mathrm{pH}$ a la coagulación, tasa de acidificación, proteólisis en agar leche, lipólisis en agar triacetina, producción de acetoína en leche reconstituida y asimilación del citrato en agar citrato. Lb. delbruekii subsp. bulgaricus, Lb. casei subsp. pseudoplantarum, $L b$. plantarum var. arabinosus, $L b$. plantarum var. plantarum, $L b$. casei subsp. rhamnosus, $L b$. acidophilus, $L b$. helveticus, $L b$. fermentum, $L b$. brevis var. brevis, Lactococos sp. y Enterococcus sp. fueron las bacterias lácticas identificadas. Del total de los aislamientos, $41,6 \%$ coagularon la leche en 10 horas y $33 \%$ en 5 horas. $L b$. helveticus coaguló la leche a pH de 5,40 en 5 horas, hasta alcanzar un valor final de 4,16 en $24 \mathrm{~h}$, mientras que $L b$. delbrueckii subsp. bulgaricus y $L b$. fermentum iniciaron la coagulación en 5 horas, con valores de pH iniciales de 4,81 y 4,92 hasta valores finales de 4,19 y 4,21 respectivamente. $L b$. helveticus, $L b$. delbrueckii subsp. bulgaricus, $L b$. plantarum var. arabinosus, $L b$. fermentum, $L b$. casei subsp. rhamnsosus, Lb. casei subsp. pseudoplantarum, $L b$. brevis var. brevis, en orden descendente, demostraron tener capacidad acidificante. Lb. fermentum y Lb. casei subsp. pseudoplantarum desarrollaron actividad proteolítica y sólo $L b$. plantarum var. plantarum demostró tener actividad lipolítica. Las levaduras aisladas fueron

Debaryomyces hansenii, Zygosaccharomyces rouxii, Kluyveromyces lactis, Wickerbamiela domerquiae, Dekkera bruxellensis, Candida valdiviana, Candida novakii, Dekkera bruxellensis, Candida versatilis, Candida magnoliae, Candida 
albicans, Pichia anómala, Dekkera anómala y Rodotorula sp. Cepas de D. hansenii, C. magnoliae, $Z$. rouxii, $C$. versatilis y $K$. lactis tuvieron actividad proteolítica y lipólitica, y una cepa de $W$. domerquiae tuvo solamente actividad proteolítica. Algunas cepas de $K$. lactis produjeron acetoína y $D$. bruxellensis y $C$. versatilis metabolizaron el citrato, hidrolizaron la caseína y tuvieron actividad lipolítica.

Los resultados obtenidos en este estudio muestran que la composición de las poblaciones de BAL y levadura en quesos artesanales es específica de la región. Los conocimientos adquiridos en este estudio podrían ser utilizados para la obtención de cultivos iniciadores con cepas de BAL y levaduras específicas de la región, destinados a la producción de quesos frescos con origen geográfico específico.

Palabras clave: quesos frescos de cabra, capacidad acidificante, actividad proteolítica, actividad lipolítica.

\section{Evaluation of the diversity of lactic bacteria and yeast in fresh goat cheese of the quebrada Humahuaca.}

\section{Abstract}

Fresh cheeses artisanal goat of the Quebrada de Humahuaca are made with raw milk, the maturation produces flavors, aromas and textures characteristic of the region.

The objectives of this study were to identify and characterize lactic acid bacteria (LAB) and native yeasts isolated from fresh cheeses productive zone. A total of 36 samples seeded on agar Sabouraud agar MRS and M17, 128 yeast and 39 lactobacilli were obtained, which were phenotypically identified and evaluated the following technological properties: $\mathrm{pH}$ of the coagulation rate of acidification, proteolysis agar milk, lipolysis in triacetin agar, acetoin production in reconstituted milk and assimilation of citrate in citrate agar. Lb. delbruekii subsp. bulgaricus, $L b$. casei subsp. pseudoplantarum, Lb. plantarum var. arabinosus, Lb. plantarum var. plantarum, $L b$. casei subsp. rhamnosus, $L b$. acidophilus, $L b$. helveticus, $L b$. fermentum, Lb. brevis var. brevis, Lactococcus sp. and Enterococcus sp. lactic acid bacteria were identified. Of all the isolates, $41.6 \%$ coagulated milk in 10 hours and $33 \%$ within 5 hours. Lb. helveticus coagulated milk to $\mathrm{pH} 5.40$ in 5 hours, to a final value of 4.16 at $24 \mathrm{~h}$, while $L b$. delbrueckii subsp. bulgaricus and Lb. fermentum initiated coagulation in 5 hours, with initial pH values of 4.81 and 4.92 up to final 


\section{5}

values of 4.19 and 4.21 respectively. $L b$. helveticus, $L b$. delbrueckii subsp. bulgaricus, $L b$. plantarum var. arabinosus, $L b$. fermentum, $L b$. casei subsp. rhamnsosus, $L b$. casei subsp. pseudoplantarum, $L b$. brevis var. brevis, in descending order, they were shown to have acidifying capacity. Lb. fermentum and $L b$. casei subsp. pseudoplantarum developed proteolytic activity and only $L b$. plantarum var. plantarum demonstrated lipolytic activity. The yeast isolates were Debaryomyces hansenii, Zygosaccharomyces rouxii, Kluyveromyces lactis, Wickerbamiela domerquiae, Dekkera bruxellensis, valdiviana Candida, Candida novakii, Dekkera bruxellensis, Candida versatilis, magnoliae Candida, Candida albicans, Pichia anomala, Dekkera anomala and Rodotorula sp. D. hansenii strains, C. magnoliae, $Z$. rouxii, $C$. versatilis and $K$. lactis had proteolytic and lipolytic activity, and a strain of $W$. domerquiae had only proteolytic activity. Some strains of $K$. lactis produced acetoin and $D$. bruxellensis and $C$. versatilis metabolize citrate, they hydrolyzed casein and had lipolytic activity.

The results obtained in this study show that the composition of the populations of BAL and baking artisan cheeses is specific to the region. The knowledge gained from this study could be used for the production of starter cultures with specific strains of $L A B$ and yeasts in the region for the production of fresh cheeses with specific geographical origin.

Keywords: fresh goat cheese, acidifying capacity, proteolytic activity, lipolytic activity.

\footnotetext{
*Para citar este articulo: Ancasi,EG; Maldonado,S; Oliszewski, R. Evaluación de la diversidad de bacterias lácticas y levaduras en quesos frescos de cabra de la quebrada de Humahuaca. Revista Bistua.2015.13(1):03-15
} 


\section{Introducción}

La Quebrada de Humahuaca, en la provincia de Jujuy, constituye una región geográfica con características propias del noroeste argentino, con una larga tradición en la producción regional de productos lácteos a través de fermentación de leche de cabra. En esta región los quesos frescos de origen artesanal, se fabrican sin el uso de cultivos iniciadores comerciales. Estos productos son elaborados con leche no pasteurizada y la composición del estárter natural depende únicamente de la microbiota presente en la leche cruda o del entorno local. Estos microorganismos son responsables del sabor, aroma y textura del queso, otorgándole características únicas al producto. Topisirovic ${ }^{1}$ informaron que las BAL aisladas de muestras de quesos autóctonos tienen un considerable potencial genético, metabólico y tecnológico a través de características como: buena coagulación de las proteínas de la leche, acidificación acelerada, producción de proteinasas, exopolisacáridos, precursores de aroma y sabor, así como las propiedades que promueven la salud. Las BAL dominantes están representadas por varias especies de lactobacilos: Leuconostocs, enterococos, Streptococcus thermophilus y Lactococcus lactis.
Las especies de levaduras generalmente representan la microbiota secundaria en quesos y entre ellos Kluyveromyces marxianus, Debaryomyces hansenii y Saccharomyces cerevisiae, entre otras, son las predominantes ${ }^{2}$.

Por otra parte, estudios recientes han indicado que levaduras de origen alimentario tales como $D$. hansenii y Yarrowia lipolytica podrían ser utilizadas como parte de los cultivos iniciadores para la manufactura de quesos, mejorando el desarrollo del sabor durante la maduración ${ }^{3}$. En relación a lo anterior el objetivo de este estudio fue caracterizar bacterias lácticas (BAL) y levaduras nativas, aisladas de quesos frescos de esta zona productora, con miras al desarrollo de un cultivo iniciador que conserve las características de los quesos locales.

\section{Materiales y Métodos Toma de Muestra}

Se tomaron 36 muestras de quesos de cabra de diferentes puntos de la Quebrada de Humahuaca de la Provincia de Jujuy, directamente de los productores. Las muestras se trasladaron inmediatamente al laboratorio en un contenedor que permitió mantener la temperatura de 
7

refrigeración. En el laboratorio las muestras se conservaron a $4{ }^{\circ} \mathrm{C}$ por un tiempo no mayor a 1 día hasta su análisis.

\section{Aislamiento e identificación de BAL}

El aislamiento de las BAL a partir de los quesos se realizó siguiendo el método según ${ }^{4}$. Para el análisis microbiológico se tomaron $25 \mathrm{~g}$ de cada muestra,se transfirió a $225 \mathrm{ml}$ de solución estéril de citrato de sodio al $2 \% \quad(w / v)$ y se homogeneizó manualmente durante 10 minutos. Se realizaron diluciones decimales y se sembraron en los medios apropiados para cada BAL: a) para lactobacilos, en agar MRS pH 5,5e incubados a 30 ${ }^{\circ} \mathrm{C}$ y $45^{\circ} \mathrm{C}$ durante $72 \mathrm{~h}$ en condiciones de microaerofilia, b) para lactococos, sobre agar M17 pH 7,2 a $30{ }^{\circ} \mathrm{C}$ durante $72 \mathrm{~h}^{5}$. Se tomó un promedio de 24 colonias al azar por muestra, de las placas de agar MRS $\left(30{ }^{\circ} \mathrm{C}\right.$ y $\left.45{ }^{\circ} \mathrm{C}\right)$ y agar $\mathrm{M} 17$, correspondientes a la dilución más alta a la que se produjo el crecimiento. La morfología de las células de todas las cepas de BAL se determinó por microscopía y se consideraron aquellos bacilos y cocos Gram positivos y catalasa negativa para su posterior análisis tecnológico e identificación según metodología propuesta por Bergey (2000). Para una correcta conservación, los lactobacilos fueron almacenados en caldo MRS $30 \%$ de glicerol (v/v) a -20 ${ }^{\circ} \mathrm{C}^{6}$.

\section{Aislamiento e identificación de levaduras}

Para el aislamiento de levaduras se tomaron porciones de $25 \mathrm{~g}$ de cada queso, los cuales fueron homogenizados en $225 \mathrm{ml}$ de solución estéril de citrato de sodio al $2 \%(\mathrm{w} / \mathrm{v})$ durante 10 minutos. De las suspensiones se realizaron diluciones decimales (10-1 a 10-6) con el mismo diluyente. De cada dilución se tomaron alícuotas de $100 \mu \mathrm{l}$ y se esparcieron en agar Sabouraud (peptona de carne 10\%, glucosa $40 \%$, agar $20 \%$ ) con cloranfenicol $(0,01 \%)$. Las placas se incubaron por 5 días a $25 \stackrel{\circ}{ } \mathrm{C}$. Transcurrido el tiempo de crecimiento se tomaron colonias elegidas en base a su morfología (superficies lisas, rugosidad de bordes, consistencia, color, etc.), repicadas en caldo YPD (1\% extracto de levadura, $2 \%$ de dextrosa y $2 \%$ de peptona) e incubadas $48 \mathrm{hs}$ a $25^{\circ} \mathrm{C}$, para su posterior análisis tecnológico e identificación, según metodología propuesta por ${ }^{7}$. Para su correcta conservación las levaduras se preservaron en caldo YPD con $20 \%$ $(\mathrm{v} / \mathrm{v})$ de glicerol. 
8

\section{Caracterización fisiológica y tecnológica de los aislados}

Las bacterias lácticas fueron pre cultivadas en caldo MRS a $30{ }^{\circ} \mathrm{C}$ durante $24 \mathrm{~h}$, para obtener 106 $\mathrm{ufc} / \mathrm{ml}$, luego sometidas a las siguientes pruebas: $\mathrm{pH}$ a la coagulación (IDF, 1980), velocidad de acidificación expresadas como $\Delta \mathrm{pH} / \mathrm{h}$ según ${ }^{8}$, capacidad lipolítica en agar triacetina y capacidad proteolítica en Agar leche descremada con la aparición de zonas transparentes alrededor de la colonia en ambos casos $^{9}$. Los aislados de levaduras fueron cultivados en caldo YPD durante 24-48 hs a 25-28 으 $y$ sometidos a las siguientes pruebas: producción de acetoína, inoculando al $0,1 \%$ tubos con $10 \mathrm{ml}$ de leche descremada reconstituida estéril $(10 \% \mathrm{P} / \mathrm{V})$, incubadas a $25-28^{\circ} \mathrm{C}$ durante 24 horas ${ }^{10}$; asimilación del citrato en agar citrato según ${ }^{11}$; la actividad proteolítica extracelular y la actividad lipolítica fue determinada según ${ }^{12}$.

\section{Resultados y Discusión}

\section{Aislamiento e identificación de BAL y levaduras}

La identificación de los aislamientos reveló la presencia de tres géneros:
Enterococcus(41,82\%), Lactobacillus $(29,40)$ y Lactococcus $(28,91)$, de los cuales se seleccionaron 39 cepas de lactobacilos. Se identificaron como bacilos Gram-positivos, catalasa negativa y se clasificaron en las siguientes especies: $L b$. delbrueckii supesp. bulgaricus (5,13\%), Lb. plantarum var. arabinosus $(23,1 \%)$, Lb. casei subesp. pseudoplantarum $(12,8 \%)$, Lb. casei subesp. rhamnosus (17,9 \%), Lb. acidophilus (23,1\%), Lb. helveticus $(5,13 \%), \quad$ Lb.fermentum $(10,3 \%)$ y $L b$. brevis (2,6\%). Lb. plantarum fue unas de las especies predominantes en las identificaciones de bacilos, lo que concuerda con trabajos similares realizados en leche de cabra ${ }^{13,14}$ demostraron la presencia de Lb.plantarum en otras variedades quesos de cabra. Mathara ${ }^{15}$ identificó a $L b$. plantarum, Lb.acidophilus, Lb. rhamnosus y Lb.fermentum en productos lácteos fermentados y puso de manifiesto que estas especies representan más del $60 \%$ de los lactobacilos aislados. Además, Medina ${ }^{16}$ demostró que el $8 \%$ de Lactobacilos aislados a partir de leche de oveja y queso en el norte de Argentina pertenecían a $L b$. acidophilus. De las 128 cepas de levaduras, las especies encontradas en un mayor porcentaje fueron Candida versatilis (13,27\%), Candida etchellsii (13,27\%), Pichia anómala $(11,50 \%)$, Debaryomyces hansenii 
9

$(8,88 \%)$, entre otras. La frecuencia de las especies restantes estuvo entre 0,88 y $6,19 \%$. Varios autores han demostrado que las levaduras prevalentes asociados típicamente con los quesos son $D$. hansenii, $K$. marxianus, Yarrowia lipolytica y varias especies de Candida ${ }^{1, .18}$.

\section{Caracterización tecnológica de los aislados.}

\section{Tasa de acidificación y pH a la coagulación de BAL}

Las pruebas de coagulación mostraron que el $100 \%$ de las cepas aisladas coagularon la leche en menos a las 24 hs; $41,6 \%$ dentro de las 10 horas y sólo el $33 \%$ dentro de las 5 horas. En orden descendente el valor de la velocidad de acidificación de las cepas aisladas fue: $L b$. helveticus $(0,094), L b$. delbrueckii subesp. bulgaricus (0,093), Lb. fermentum $(0,092)$, Lb. plantarum var. arabinosus $(0,0921), \quad L b$. casei subesp. rhamnsosus $(0,091), L b$. casei subesp. pseudoplantarum $(0,085)$, Lb. brevis var. brevis $(0,08)$. Este parámetro es considerado un factor fundamental en la elección de cepas iniciadoras, el que asegura que los quesos tengan un rápido descenso de $\mathrm{pH}$ y una adecuada degradación enzimática de la caseína $^{19}$. La capacidad de acidificación, expresada como $\mathrm{pH}$ de la leche al momento de la coagulación, mostró que $L b$. helveticus provocó un $\mathrm{pH}$ de 5,40 a las 5 horas, llegando a un $\mathrm{pH}$ final de 4,16 a las 24 hs; en tanto que $L b$. delbrueckii subesp. bulgaricus y $L b$. fermentum produjeron la coagulación de la leche a las 7 horas, con valores de $\mathrm{pH}$ de 4,81 y 4,92, obteniendo valores finales de $\mathrm{pH}$ de 4,19 y 4,21 respectivamente. Algunas de las especies estudiadas son similares a las obtenidas por ${ }^{19,20}$.

\section{Actividad proteolítica de BAL y levaduras}

Entre las cepas que demostraron tener esta actividad se encuentran las siguientes: Lb. delbrueckii supesp. bulgaricus (2 cepas), Lb.casei subesp. pseudoplantarum (5 cepas), Lb. plantarum var. arabinosus (4 cepas), Lb. casei subesp. rhamnosus ( 2 cepas), Lb. acidophilus (6 cepas), Lb. helveticus (1 cepa), Lb. brevis var. brevis (1 cepa) y Lb. fermentum (3 cepas). Sin embargo, se observaron diferencias en la eficiencia de la hidrólisis de la caseína, siendo $L b$. fermentum (1 cepa) la que obtuvo una zona clara de $7 \mathrm{~mm}$ de diámetro, Lb.casei subesp. pseudoplantarum (1 cepa) con $6,3 \mathrm{~mm}$ y $L b$. brevis var. brevis (1 cepa) con 6,2 mm entre las cepas más relevantes. La actividad 
proteolítica de $L b$. casei y $L b$. plantarum concuerda con los resultados obtenidos por ${ }^{21}$ Herrero (2003). Settanni ${ }^{22}$ demostró que $L b$. brevis y $L b$. plantarum, dos especies clasificadas como NSLAB (Non Starter Lactic Acid Bacteria), con poca actividad proteolítica pueden poner en manifiesto el desarrollo de sabor en quesos. Entre las levaduras aisladas presentó mayor actividad proteolítica Debaryomyces hansenii (4 cepa; $5 \mathrm{~mm}$ ) seguida por que Wickerbamiella domerquiae (1 cepa; $3,8 \mathrm{~mm}$ ), Zygosacharomyces rouxii (2 cepas; $3,5 \mathrm{~mm}$ ), Cándida magnoliae ( 2 cepas; $3 \mathrm{~mm}$ ), Pichia canadensis (1 cepa; $2,5 \mathrm{~mm}$ ) y Kluyveromyces lactis ( 2 cepas; $2 \mathrm{~mm}$ ). La actividad proteolítica de la especie $D$. hansenii es variable. Numerosos autores informaron la ausencia de esta especie ${ }^{23,24}$, mientras que estudios recientes de ${ }^{25,26}$, determinaron la actividad proteolítica intracelular en cepas aisladas de quesos.

Recientemente, se ha demostrado que algunas especies de levaduras, tales como $D$. hansenii y K.lactis, con frecuencia aisladas de quesos, pueden formar péptidos biológicamente activos (ChavesLópez et al., 2012) o con actividades antioxidantes y antigenotóxicas ${ }^{27}$.

\section{Actividad Lipolítica de BAL y levaduras}

10

En este estudio, la actividad lipasa extracelular se exhibió por sólo unas pocas cepas $L b$. plantarum var. arabinosus ( 2 cepas), mientras que solo el $14,2 \%$ de las cepas de levaduras presentaron lipolisis. De estas cepas las que obtuvieron mayor actividad fueron Dekkera bruxellensis (4 cepas, $4 \mathrm{~mm}$ ), Pichia canadensis (3 cepas, 3mm), Debaryomyces hansenii ( 2 cepas, $5 \mathrm{~mm}$ ) y Pichia anómala (1 cepa, $2 \mathrm{~mm}$ ). Suzii y colaboradores (2001) han informado sobre la alta actividad lipolítica de las levaduras y su contribución a la maduración del queso. La especificidad de la lipasa y el posterior metabolismo de los ácidos grasos libres son importantes para la caracterización aromática de los quesos. El olor característico de los quesos frescos puede provenir de la fracción lipídica y de la cantidad de ácidos grasos libres, el ácido 4metiloctanoico y el ácido 4etiloctanoico pueden ser los principales responsables de esta característica $^{28}$.

\section{Producción de acetoína y asimilación del citrato de levaduras}

La mayoría de las cepas mostraron capacidad para fermentar la glucosa $(42,5 \%)$, Las levaduras identificadas que presentaron mayor porcentaje de 
esta actividad fueron Candida versatilis, Pichia anómala y Candida lactis-condensi, las que fueron capaces de asimilar y fermentar la lactosa. La asimilación de lactato se observó en la mayoría de las cepas, mientras que la asimilación de citrato fue una característica de Candida etchellsii, y Candida novakii. Alternativamente, hay un gran interés en la posibilidad de la producción de ácido cítrico por diferentes cepas de levadura. Las levaduras pertenecientes a especies Yarrowia (syn. Candida, Saccharomycopsis, Endomycopsis) lipolytica, C. guillermondii y $C$. oleophila son conocidas por ser capaces de producir un amplio espectro de ácidos orgánicos, incluidos los intermediarios del ciclo tricarboxílico, tales como ácido cítrico y ácido isocítrico, a partir de una amplia gama de fuentes de carbono, en particular, los n-alcanos, glucosa, glicerol, etanol y galactosa ${ }^{29}$. El citrato al igual que la producción de acetoina, son precursores importantes para los compuestos de sabor en ciertas variedades de quesos ${ }^{30}$.

\section{Conclusiones}

La evaluación de la diversidad de BAL y de levaduras obtenidas en este estudio pone de manifiesto su fuerte influencia en los procesos tradicionales de producción y en las características organolépticas de los quesos regionales. Las cepas seleccionadas en base a su velocidad acidificante y capacidad de coagulación, tales como $L b$. helveticus, Lb.delbrueckii subesp. bulgaricus y $L b$. fermentum, podrían ser utilizadas como cepas iniciadoras, mientras que, Lb. casei subesp.pseudoplantarum y $L b$. plantarum var. arabinosus demostraron tener una buena actividad como co-iniciadores para la fabricación de quesos. En cuanto a las especies de levadura, fueron diversas y específicas de la zona. En particular, considerando que algunas cepas de Debaryomyces hansenii, Dekkera bruxellensis, Candida versatilis y Candida etchellsii, que proporcionaron características tecnológicas deseables como proteólisis, lipolisis y producción de acetoina, pueden ser utilizadas como parte de un estárter destinado a la producción de quesos frescos con origen geográfico específico.

\section{Referencias bibliográficas.}

1.-Topisirovic, L., Kojic, M., Fira, D., Golic, N., Strahinic, I. and Lozo, J. Potential of lactic acid bacteria isolated from specific natural niches in food production and preservation. International Journal of Food Microbiology. 2006; 112: 230-235. 
12

2.-Chebenová-Turcovská, V., Zenišová, K., Kuchta, T., Pangallo, D. and Brežná, B. Culture independent detection of microorganisms in traditional Slovakian bryndza cheese. International. Journal of Food Microbiology 2011; 150: 73-78..

3.-Sørensen, L.M., Gori, K., Petersen, M.A., Jespersen and L., Arneborg, N. Flavour compound production by Yarrowia lipolytica, Saccharomyces cerevisiae and Debaryomyces hansenii in a cheese-surface model. International Dairy Journal 2011; 21: 970-978.

4.-Terzic-Vidojevic, A., Vukasinovic, M., Veljovic, K., Ostojic, M. and Topisirovic, L. Characterization of microflora in homemade semi-hard white Zlatar cheese. International Journal of Food Microbiology. 2007; 14: 36-42.

5.-Mannu, L., Riu, G., Comunian, R., Frozzi, M.C., Scintu, M.F. A preliminary study of lactic acid bacteria in whey starter culture and industrial Pecorino Sardo ewes, milk cheese: PCR-identification and evolution during ripening. International Dairy Journal. 2002; 12: 17-26.
6.-Bergey, \& Holt, John G Bergey's manual of determinative bacteriology (9th ed). Lippincott, Williams \& Wilkins, Baltimore. (2000).

7.-Barnett, JA, Payne RW \& Yarrow D. Yeast's characteristics and Identification. Third Edition. United Kingdom. Cambridge University Press. 2007

8.-Chaves-López, C., Tofalo, R., Serio, A., Paparella, A., Sacchetti, G. and Suzzi, G. Yeasts from Colombian Kumis as source of peptides with Angiotensin I converting enzyme (ACE) inhibitory activity in milk. International Journal of Food Microbiology. 2012; 159: 39-46.

9.-Moulay, M., Aggad, H., Benmechernene, Z., Guessas, B., Henni, D.E. and Kihal, M. Cultivable Lactic Acid Bacteria Isolated from Algerian Raw Goat's Milk and Their Proteolytic Activity. World Journal of Dairy \& Food Sciences 2006; 1 (1): 12-18.

10.-Mac Faddin, J.F. Pruebas bioquímicas para la identificación de bacterias de importancia clínica. Editorial Médica Panamericana. 1980.

11.-Suzzi, G., Lanorte, M. T., Galgano, F., Andrighetta, C., Lombardi, A., Lanciotti, R. \& 
13

Guerzoni,M. E. Proteolytic, lipolytic and molecular characterization of Yarrowia lipolítica isolated from cheesell. International Journal of Food Microbiology. 2001; 69: 69-77.

12.-Kurtzman, C.P., Fell, J.W., Boekhout, T., Robert, V. Methods for isolation, phenotypic characterization and maintenance of yeasts, 5th edn. In: Kurtzman, C.P., Fell, J.W., Boekhout,T. (Eds.), The Yeasts, a Taxonomic Study. Elsevier, Amsterdam 2011; vol. 1.: pp. 87-110.

13.-Badis A, Guetarni D, Boudjema MB, Henni DJ \& Kihal M. Identification and technological properties of lactic acid bacteria isolated from raw goat milk of four Algerian race. Food Microbiol. 2004; 21: 579-588.

14.-Olarte, C., Sanz, S., GonzálesFandos, E., Torre, P. The effect of a commercial starter culture addition on the ripening of an artisanal goat's cheese (Cameros cheese). Journal of Applied Microbiology. 2000; 88: 421429.

15.-Mathara JM, Schillinger U, Kutima PM, Mbugua SK, Holzapfel WH. Isolation, identification and characterization of the dominant microorganisms of kulenaoto: the Maasai traditional fermented milk in
Kenya. Int J Food Microbiol 2004; 94:269-278.

16.-Medina, R.; Katz, M.; González, S.; Oliver, G. Characterization of the lactic acid bacteria in ewe's milk and cheese from Northwest Argentina. Journal of Food Protection. 2001; 64: 559-563.

17.-Borelli, Beatriz M., Elaine G. Ferreira, Inayara C. A. Lacerda, Glória R. Franco, Carlos A. Rosa. Yeast populations associated with the artisanal cheese produced in the region of Serra da Canastra, Brazil. World Journal of Microbiology and Biotechnology. November. 2006. Volume 22, Issue 11, pp 1115-1119.

18.-Mehlomakulu, Ngwekazi Nwabisa. Yeasts as adjunct starter cultures in cheese making. Tesis magister. Faculty of Natural and Agricultural Sciences. Department of Microbial, Biochemical and Food Biotechnology. University of the Free State. Bloemfontein. 2011.

19.-Oliszewski, R. Leche de cabra: su calidad y evaluación de starters lácticos caprinos regionales en la manufactura de productos fermentados. Tesis doctoral. Universidad Nacional de Tucumán. 2006. 
14

20.-Cheriguene A, Chougrani F, Bekada AMA, El Soda M, Bensoltane A. Enumeration and identification of lactic microflora in Algerian goat's milk. Afr. J. Biotechnol. 2007; 6(1 5): 1854-1861.

21.-Herreros, M.A, J.M Fresno, M.J González Prieto, M.E Tornadijo. Technological characterization of lactic acid bacteria isolated from Armada cheese (a Spanish goats'milk cheese).International Dairy Journal. 2003; Volume 13, Issue 6, Pages 469-479.

22.-Settanni, L. and Moschetti, G. Non-starter lactic acid bacteria used to improve cheese quality and provide health benefits. Food Microbiology 2010; 27: 691-697..

23.-Welthagen, J. J. \& Viljoen, B. C. The presence of yeasts in different cheese types. In Yeasts in the dairy industry: positive and negative aspects, Jakobsen, M., Narvhus, J., Viljoen,B.C. (Eds.), Copenhagen, Denmark. International Dairy Federation, Brussels, Belgium. 1998; 78-87.

24.-Van den Tempel, T.and Jakobsen, M. The technological characteristics of Debaryomyceshansenii and
Yarrowialipolytica and their potential as starter cultures for production of Danablu. International Dairy Journal.2000; 10(4): 263-270.

25.-Klein, N., Zourari, A., Lortal, S. Peptidase activity of four yeast species frequently encountered in dairy products and comparison with several dairy bacteria. International Dairy Journal 2002; 12: 853-861.

26.-Bintsis, T.; VafopoulouMastrojiannaki, A.; LitopoulouTzanetaki, E. and Robinson, R.K. Protease, peptidase and esterase activities by lactobacilli and yeast isolates from Feta cheese brine. Journal of Applied Microbiology 2003; 95: 68-77.

27.-Trotta, F., Caldini, G., Dominici, L., Federici, E., Tofalo, R., Schirone, M., Corsetti, A.Suzzi,G. and Cenci, G. Food borne yeasts as DNAbioprotective agents against model genotoxins. International Journal of Food Microbiology. 2012; 153: 275280.

28.-Gaborit, P., Menard A., Morgan F. Impact of ripening strains on the typical flavour of goat cheeses. International Dairy Journal 2001;11: 315-325. 
15

29.-Kamzolova, S.V., Morgunov, G.I., Aurich, A., Perevoznikova, O.A., Shishkanova, N. V.,Stottmeister, U. and Finogenova, T.V. Lipase Secretion and Citric Acid Production in Yarrowia lipolytica Yeast Grown on Animal and Vegetable Fat. Food Technol. Biotechnol. 2006; 43 (2): 113-122.

30.-Fox P.F. Cheese overview II. Academic press, London. 2002.

Ancasi,EG. M.Sc. Facultad de Ciencias Agrarias. Universidad Nacional de Jujuy. Alberdi 47- (4600). San Salvador de Jujuy. Jujuy. Argentina. Email: gustyancasi@hotmail.com.

Maldonado,S .MSc. Facultad de Ciencias Agrarias. Universidad Nacional de Jujuy. Alberdi 47- (4600). San Salvador de Jujuy. Jujuy. Argentina.

Oliszewski, R.CONICET - Fac. Agronomía y Zootecnia. (4000). Universidad Nacional de Tucumán.Tucumán. Argentina. 